\title{
Design and Implementation of a Rural Social Security System Based on Deep Learning
}

\author{
Yan Zhao \\ School of Economics and Management, Inner Mongolia Normal University, Hohhot, 010022 Inner Mongolia, China \\ Correspondence should be addressed to Yan Zhao; zhaoyan@imnu.edu.cn
}

Received 27 May 2021; Revised 8 July 2021; Accepted 26 July 2021; Published 26 August 2021

Academic Editor: Wenqing Wu

Copyright ( 2021 Yan Zhao. This is an open access article distributed under the Creative Commons Attribution License, which permits unrestricted use, distribution, and reproduction in any medium, provided the original work is properly cited.

\begin{abstract}
After the reform and opening up, my country's economic level and total national strength have achieved unprecedented growth. The building of a well-off society in an all-round way is moving towards a harmonious society. The development of social security is also an important part of the development and improvement of a socialist harmonious society. This article is aimed at designing a rural social security system based on deep learning algorithms, using sample collection and statistical analysis methods, collecting samples, simplifying the algorithm, and establishing a new rural social security system. The data collected by the system shows that the proportion of farmers who choose very satisfied, satisfied, average, dissatisfied, and very dissatisfied with the satisfaction of the new rural insurance is $8.94 \%, 45.53 \%, 34.96 \%, 8.13 \%$, and $2.44 \%$. It can be seen that the proportion of farmers who choose to be satisfied is the largest, and more than $10.0 \%$ of farmers choose to be dissatisfied or very dissatisfied. Investigate the factors that farmers worry about participating in the new rural insurance, and the questionnaire options can also be set to multiple choices. The survey results show that $29.27 \%$ of the farmers think that the individual payment for participating in the new rural insurance is higher; $26.02 \%$ of the farmers believe that they do not understand the new rural insurance system; $9.76 \%$ of the farmers believe that it is unnecessary to pay for the new rural insurance; $22.76 \%$ of farmers choose to rely on themselves or their children in the future; $27.64 \%$ of farmers think that the system is unstable. It has basically realized the design of a brand new rural social security system starting from the deep learning of semantic computing.
\end{abstract}

\section{Introduction}

The rural social security system (the new rural endowment insurance system is a personal endowment insurance account in which the state establishes a lifetime record for each new rural insurance participant; individual payment, collective subsidies, and other economic organizations, social welfare organizations, and individual subsidies for the insured person's payment, and the local government's subsidy for the insured person's payment, all are credited to the personal account) is an indispensable system of people's livelihood security proposed based on our country's social status quo. Nowadays, most countries and regions are facing the problem of aging population. The issue of aging has increasingly become a hot issue of concern to various countries. At this stage, my country has become one of the countries with the largest aging population. Data shows that my country's aging population will reach its peak in the middle of this century, and my country's aging problem will be extremely important. Through comparison between the urban and rural areas, the problem of rural population aging is even more serious. Therefore, solving the problem of the aging of the rural population is an important step in solving the problem of the aging of the population in our country. For a long time, my country's dual economy has caused the rural economy to lag far behind the urban areas. For example, rural areas are at a disadvantage compared with urban areas in terms of pension insurance. Nowadays, the majority of rural residents are not fully guaranteed for the elderly, and the concept of raising children and preventing the elderly is still deeply ingrained in many rural areas. With the continuous development of the national economy, the outflow of agricultural laborers and the development of family planning have resulted in the labor force being less than the elderly population. These undoubtedly increase the burden of agricultural pensions. Therefore, there is an urgent need to solve the problem of agricultural pensions. No matter from a theoretical level 
or a practical point of view, it is imperative to solve the problem of rural old age security. In theory, the establishment of a modern old age security system in the vast rural areas can better enhance farmers' awareness of old age security and enrich my country's overall old age security system. From a practical point of view, it can effectively solve the current problem of providing services for the elderly in the vast rural areas of our country. At the same time, solving the problem of rural endowment insurance is an important way to develop the rural economy, build a new socialist countryside, and build a harmonious society. It is the only way to realize the Chinese people's pension in rural areas.

Deep learning is an important part of the field of artificial intelligence research. Machine learning allows computers to learn. Without the participation of machine learning, artificial intelligence will become insufficient. Therefore, machine learning is essential to the field of artificial intelligence. Through learning algorithms, machine learning enables the machine to learn the internal laws of the data from a large amount of data input from abroad so that it can identify and judge. The development of machine learning has experienced two major development stages of shallow learning and deep learning in history. Generally, deep learning can be understood as an important development of artificial neural networks. The artificial neural network (the artificial neural network is a research hotspot that has emerged in the field of artificial intelligence since the 1980s; it abstracts the human brain neuron network from the perspective of information processing, establishes a certain simple model, and composes different networks according to different connection methods; in engineering and academia, it is often abbreviated as the neural network or quasi-neural network; the aneural network is a computing model, which is composed of a large number of nodes (or neurons) connected to each other; each node represents a specific output function, called an activation function; each connection between two nodes represents a weighted value for the signal passing through the connection, which is called a weight, which is equivalent to the memory of an artificial neural network; the output of the network varies according to the connection method of the network, the weight value, and the activation function; the network itself is usually an approximation of a certain algorithm or function in nature, or it may be an expression of a logic strategy) simulates the neural network of the human brain to remove features and model it, learn from the knowledge imported from the external environment, and have the recognition and judgment capabilities similar to the animal brain. The artificial neural network is an important part of intelligence, and it provides an effective way to solve complex problems and control intelligence. Artificial neural networks are also an important research direction in the field of machine learning. Based on the deep learning method, this paper has determined the research goal, simplified the complex algorithm and designed the software, and realized the design and realization of the rural social security system. Deep learning applications include speech recognition, automatic machine translation, instant visual translation (photograph translation), and self-driving cars, which involve the intelligent direction of semantic computing.
In 1986, Wu et al. published an article on the application of the backpropagation algorithm, which is still in use today. But their algorithm is complex and general, and they did not design a more concise and simplified formula [1]. In 1998, Chen and Smys developed the LeNet5 convolutional neural network model. The network model contains 2 convergence layers, 2 concentrated layers, and 2 fully connected layers. This method uses local connection and weight distribution methods, which not only extracts features from the data but also reduces a large number of weight parameters. However, due to the limitation of computing resources at that time, even training a small neural network took a long time. Compared with other classification models in the same period (such as supporting carrier machines), it has no major advantages. In addition, when using the backpropagation algorithm on the traditional neural network, it is easy to deal with the problem of the disappearance of the step size [2]. In 2006, Zhong et al. published an article on deep neural networks in Science and proposed the idea of deep learning algorithms, which promoted the development of artificial neural networks and the application of deep learning methods in the field of image recognition. However, their research focuses on image recognition rather than optimization algorithms, and the proposed algorithm is still not easy to understand and not easy to operate [3].

The innovations of this article are as follows. (1) Under the general trend of vigorous development of intelligence, pioneeringly combine the concept of deep learning, a semantic computing aspect, with the rural social security system and consider rural society from a new perspective. Under the background of rural households' livelihood transformation, that is, under the separation of production and life of rural households, starting from the perspective of rural households, constructing rural household decision-making models to provide theoretical support for the improvement of a rural social security system is beneficial to rural economic development and rural stability. Sustainable development of the social security system guarantees this basic livelihood issue. (2) The algorithm that simplifies the training process of the convolutional neural network and the gradient descent method is studied. Compared with the traditional complex algorithm, it is more concise, and it is convenient to establish a model related to the actual problem. (3) The output efficiency of the system is maintained at a very high level. At the same time, a series of relevant government policies have been added to the algorithm to strictly ensure the standardization and efficiency of the system.

\section{Design and Implementation Method of a Rural Social Security System Based on Deep Learning}

2.1. Rural Social Security. The security system in which the state and social organizations provide financial and material assistance to all farmers in accordance with the law is called agricultural social security [4]. Its purpose is to narrow the gap between rich and poor among members of society, gradually improve the material life of farmers, and ensure social 
security [5]. Fair and stable, realize social stability and economic development in rural areas, provide a good environment for rural development, promote healthy and sustainable development in rural areas, and accelerate agricultural modernization [6]. My country's rural social security system includes the following parts: rural social security, rural social welfare, rural social assistance, and rural preferential treatment. Agricultural social insurance covers a wide range of fields, such as family planning insurance, occupational injury insurance, medical insurance, and pension insurance [7]. However, the aging of the rural population in our country is extremely serious, the population is relatively dense, and farmers are unable to carry out their daily lives due to medical treatment, which increase the burden on farmers [8]. Therefore, my country's current agricultural social security system mainly includes aging and medical care, with the focus on the construction of the rural social pension and cooperative medical system [9]. Agricultural cultural relics insurance refers to the provision of pensions to farmers who have reached the retirement age in accordance with relevant laws and regulations of our country [10]. The purpose is to ensure the basic livelihood of retired farmers. The pension fund is jointly funded and established by the state, enterprises, and individuals [11]. Rural cooperative medical care is an interactive medical insurance system supported by the government. It is jointly funded by rural economic organizations and rural groups to reduce rural poverty caused by diseases and protect the health of rural residents [12].

Rural social assistance refers to a system in which the state and social groups provide material assistance to farmers who cannot guarantee their daily lives due to special reasons [13]. The system is aimed at maintaining income loss or income that cannot meet the minimum living needs of farmers [14]. At present, my country's rural social assistance focuses on disaster relief and rural livelihood systems [15]. The target of disaster relief is natural disaster farmers, and the target of the minimum agricultural security system is poor farmers who have not yet solved their clothing deficiencies [16]. The prosperity of rural society is a public welfare activity, which is a guarantee provided to certain special farmer groups, such as the elderly and the disabled [17], as well as vulnerable groups in rural areas. Its main purpose is to provide financial and material assistance to the elderly who cannot take care of themselves and the elderly without children in rural areas to ensure their basic living standards [18]. At the same time, they provide mental and physical support to the elderly, widows, and disabled in rural areas [19]. Help improve the living standards of rural residents [20]. In recent years, the per capita income and consumption levels of Chinese farmers have undergone earth-shaking changes [21]. At the same time, the original level of social welfare has been unable to meet their current needs [22]. Former farmers pay more attention to their living standards, such as food, clothing, housing, and transportation [23]. Now, they have begun to pay attention to their spiritual consumption needs [24]. However, rural social welfare security requires higher capital investment from state finance, which is extremely difficult for a developing country with a large population, so social welfare in rural areas cannot be realized in the short term [25].
2.2. Convolutional Neural Network. In the field of deep learning, the most widely used convergent neural network (also known as convergent network) is a neural network specifically designed to process data with a similar grid structure. Compact neural networks perform well in many application areas, such as time series data (which can be thought of as a onedimensional grid formed regularly over time) and image data (which can be regarded as a two-dimensional grid of pixels) [26-27]. A trainable multilayer network structure is composed of multiple single-layer convolutional neural networks. In convergent network terminology, the first parameter of convergence is usually called the input (that is, the data to be processed), the second parameter is called the kernel function (can be regarded as the processing function), and the output is called the feature map. In a traditional neural network, the output of a neuron is related to the weight of the input node and its connection, but the weight will not be reused according to the calculation rules of the calculation type; that is, when multiplied by an element, the input will not be used again. Parameter sharing exists in the feature map. Each feature map in level 2 will detect the same feature map in level 1. The same idea is expressed as a network with bound weights (i.e., weight distribution). The specific weight sharing is shown in Figure 1.

A typical plane in a convergent neural network includes three stages. In the first stage, the layer computes multiple convergences in parallel to generate a set of linear activation responses. In the second stage, each linear activation response will pass through a nonlinear activation function. This phase is sometimes called the detection phase. In the third stage, the concentrated function provided by the cohesive neural network is again used to optimize its output. The centralized function is responsible for processing the element's output from the adhesive layer to replace the network output at this location. The use of centralized functions can be considered a sufficiently powerful additional function before: the functions obtained through this level of learning must have a small amount of variable translation requirements. When this assumption is true, concentration can significantly improve the statistical performance of the network [28].

2.3. Simplification of the Algorithm. The training of convolutional neural networks can be mainly classified into two algorithms, the gradient descent method and the backpropagation method. This part will simplify the algorithm of the gradient descent method.

The whole process takes the pixel data of the picture as input and extracts different features through several convolutional layers. Each convolutional layer is followed by a pooling layer to reduce the number of parameters, and finally, the fully connected layer is used to calculate the loss function, as shown in

$$
\begin{array}{r}
Z=W^{T} X, \\
h(x)=g(z) .
\end{array}
$$

Among them, $X$ refers to the pixel data matrix of the image and $W^{T}$ refers to the transposition of the weight 


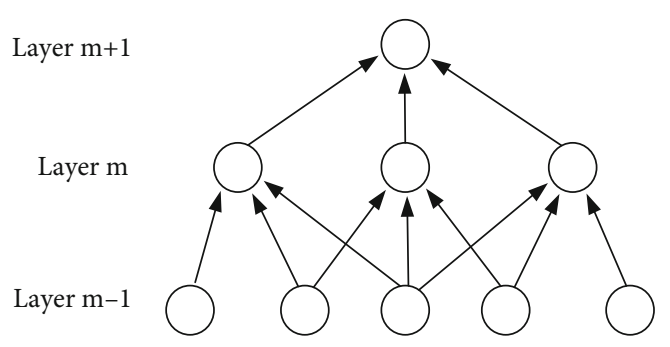

FIgURE 1: Weight sharing.

parameter matrix, which is the same as the input dimension, which contains a bias vector and the parameter weight of each data feature. The two are multiplied and then passed through. The activation function of $g$ is used as the output of this layer, $g$ can be an activation function such as sigmoid function or ReLU, and the output obtained can be used as the neuron input value of the next layer of the network, until the output result of the last layer is compared with the actual result. Construct a loss function, as shown in

$$
C(h, X, Y)=\cos t(h(x), Y)=\frac{1}{2}(h(x)-y)^{2} .
$$

Among them, $X$ represents the input, $h(x)$ represents the output value after the last layer is activated, and $Y$ represents the actual output of the input data sample. The cost function is a loss function, generally a square loss function. The $C(h$, $X, Y)$ obtained by the equation is the error value between the result and the label. When the error value continues to decrease, it means that the neural network model makes smaller and smaller errors. Based on the above requirements, it is possible to obtain the partial derivative of the weight of the equation and use the partial derivative to continuously update the weight parameter, which is the principle of the gradient descent method. Equation (4) is the partial derivative formula of this parameter:

$$
\frac{\partial C(\theta)}{\partial \theta}=\lim _{\delta \theta \longrightarrow 0} \frac{C(\theta+\delta \theta)}{\delta \theta}=-\frac{1}{m} \sum_{i=1}^{m}\left(y^{i}-h_{\theta}\left(x^{i}\right)\right) x^{j},
$$

where $\theta$ represents each weight parameter, and the obtained partial derivative is used as the adjusted value of the weight parameter, as described in

$$
\theta^{k+1}=\theta^{k}-\eta \frac{\partial C\left(\theta^{k}\right)}{\partial \theta^{k}}
$$

Since there are so many weight parameters in the network, the calculation of partial derivatives for each parameter will lead to a huge amount of calculation, and the use of the backpropagation method can solve the problem of a large amount of calculation. The basic idea of the algorithm is that the residual of the latter layer is formed by the residual of the previous layer. Therefore, the residual of the previous layer can be obtained by finding the residual of the last layer, as shown in

$$
\delta_{i}^{(n l)}=\frac{\partial C}{\partial z_{i}^{(n l)}}=\left(a_{i}^{(n l)}-y_{i}\right) \cdot f^{\prime}\left(z_{i}^{(n l)}\right) .
$$

It can be seen that the residual value of the neuron is actually the partial derivative of the weighted input of the loss value to this layer of the neuron. Then, for the number of layers $l$, the residual value should be as shown in Equation (7) below:

$$
\delta^{l}=\left(W^{l+1}\right)^{T} \delta^{(l+1)} * g^{\prime}\left(z^{l}\right)
$$

Derive the following partial derivatives of the weight parameters (in the quality evaluation process, in order to be able to express the extent to which the relevant inspection items meet the specified requirements with data, according to the amount of work occupied by each item and the degree of importance of affecting the overall ability, the proportion of each item is divided (value)) and bias vectors in each neuron, as shown in

$$
\begin{gathered}
\frac{\partial C}{\partial w_{i j}^{(l)}}=a_{j}^{(l-1)} \delta_{i}^{(l)}, \\
\frac{\partial C}{\partial b_{i}^{(l)}}=\delta_{i}^{l} .
\end{gathered}
$$

According to the above two formulas, multiply the learning rate to update each weight parameter and bias vector until the error value of the loss function is small enough.

In actual research and application, what we need to get is the distribution of observation data $v$ defined by RBM, that is, the marginal distribution of common probability distributions, also called probability function distributions, as shown in Equation (10) (marginal distribution refers to the probability distribution of only part of the multidimensional random variables in probability theory and statistics):

$$
P(\nu, \theta)=\frac{1}{Z(\theta)} \sum_{h} e^{-E(v, h \mid \theta)} .
$$

The offset $a_{i}$ of the visible layer unit is expressed as

$$
\frac{\partial \log P(v \mid \theta)}{\partial a_{i}}=\left\langle v_{i}\right\rangle_{\text {data }}-\left\langle v_{i}\right\rangle_{\text {model }}
$$

The partial derivative of the bias $b_{j}$ of the hidden layer unit is expressed as

$$
\frac{\partial \log P(v \mid \theta)}{\partial b_{i}}=\left\langle h_{i}\right\rangle_{\text {data }}-\left\langle h_{i}\right\rangle_{\text {model }}
$$

In summary, we have completed the algorithm optimization of the gradient descent method of the convolutional neural network. 


\section{Design and Implementation Method Experiment of a Rural Social Security System Based on Deep Learning}

\subsection{System Requirement Analysis}

3.1.1. Functional Requirement Analysis. The main function of this research system is to complete the storage of income and expenditure, business processing, and generation of various files of the municipal, county, district, and township and rural social endowment insurance funds. (One is the livelihood status of farmers, from the perspective of farmers' arable land area, income sources, main expenditures, and difficulties faced in production and life. The second is to investigate the implementation status of the rural social insurance system from the perspectives of farmers' participation in the new rural social pension insurance and new rural cooperative medical insurance, payment levels, and policy satisfaction. Third, the evaluation of the implementation effect of the rural minimum living security system is carried out from the perspectives of farmers' satisfaction with the system, the minimum living security standard, and the effect of poverty reduction.) With municipal management as the center, a central database is established to supervise, manage, and analyze data. Take county-level management as the main body, and carry out the management of various businesses and townships as auxiliary management. Most business operations can be carried out when the conditions are ripe.

The city bureau network version of this system mainly completes the business module design including township (village) management, personnel participation management, insurance relationship change management, payment management, account management, benefit distribution management, system connection management, financial management, financial subsidy management, and comprehensive query management, as well as information publicity, statistical reports, and system maintenance. No matter what kind of software the server is running on, the outlets can use the above Win7 operating system. The city level can operate the data of the city. Each county and city can only use and operate the data of the county and city. Each town can only use and operate the data of the county and city and can use and manipulate the data of the township.

3.1.2. Analysis of Nonfunctional Requirements. Since the proposal and final application of this topic provide a usable army equipment management system for specific units, it is necessary to consider how to build a stable, easy-to-maintain, expandable, and highly supportive system based on actual usage (concurrent number of troops of the equipment management system).

The construction of the server-side environment is one of the research contents of this topic. As a server, the machine is required to have enough loads for multiple users to access the required hardware configuration at the same time and have enough hard disk space to store data, and the machine is required to be equipped with the software interface system required by the operating system. In addition, it is necessary to combine the existing network conditions of the internal network, comprehensively consider factors such as stability, concurrency, security, and ease of use, and conduct reasonable selection, configuration, testing, evaluation, and evaluation of web services, databases, and operating systems. Trial deployment gets a strong operating, stable performance server-side environment construction plan.

3.2. The Data Flow of the System. Next, we design the data flow of the system.

The data flow diagram illustrates the information flow and data conversion from input to output. It is the logical process of data flow and processing in software and is the analysis of flow, storage, transmission, processing, and information. When designing a flowchart, only the basic operating logic of the system should be considered, not how to implement these functions in detail. The data processing of agricultural social insurance activities is basically divided into 7 parts. First, participate in the insurance and create file information based on the declared initial personal information. Then, pay the fee, and based on the file information, generate the payment information according to the declared payment details. Follow up payment account opening, according to the declared account opening personnel list, and based on the existing payment information, generate the issuance standard. According to the designated issuance time period, on the basis of the existing issuance standards, the issuance information is generated. According to the declared list of final insurance personnel, the final insurance information is generated by referring to the issuance information and payment information. According to the transfer-out application, the transfer-out information is generated on the basis of the existing file information, and the file information is modified. Finally, according to the surrender application, refer to the payment information to generate surrender information.

3.3. System Business Design. This system meets the needs of the daily work of the new rural social endowment insurance management (the survey adopts a random sampling method; the survey content mainly covers three aspects: first, the production and living conditions of farmers, from the perspective of farmland area, income sources, main expenditures, and difficulties faced by farmers in production and life; the second is to investigate the implementation status of the rural social insurance system from the perspectives of farmers' participation in the new rural social endowment insurance and new rural cooperative medical insurance, payment levels, and policy satisfaction; and third, the evaluation of the implementation effect of the rural minimum living security system is carried out from the perspectives of farmers' satisfaction with the system, the minimum living security standard, and the effect of poverty reduction), with complete functions, easy operation, and practical management software. Through investigation, I learned about the business related to the daily work of the rural social pension insurance management department. The business of the system consists of the following parts. Next, we will introduce several important business processes in the system design of this article.

The business flowchart of insurance registration is shown in Figure 2. 

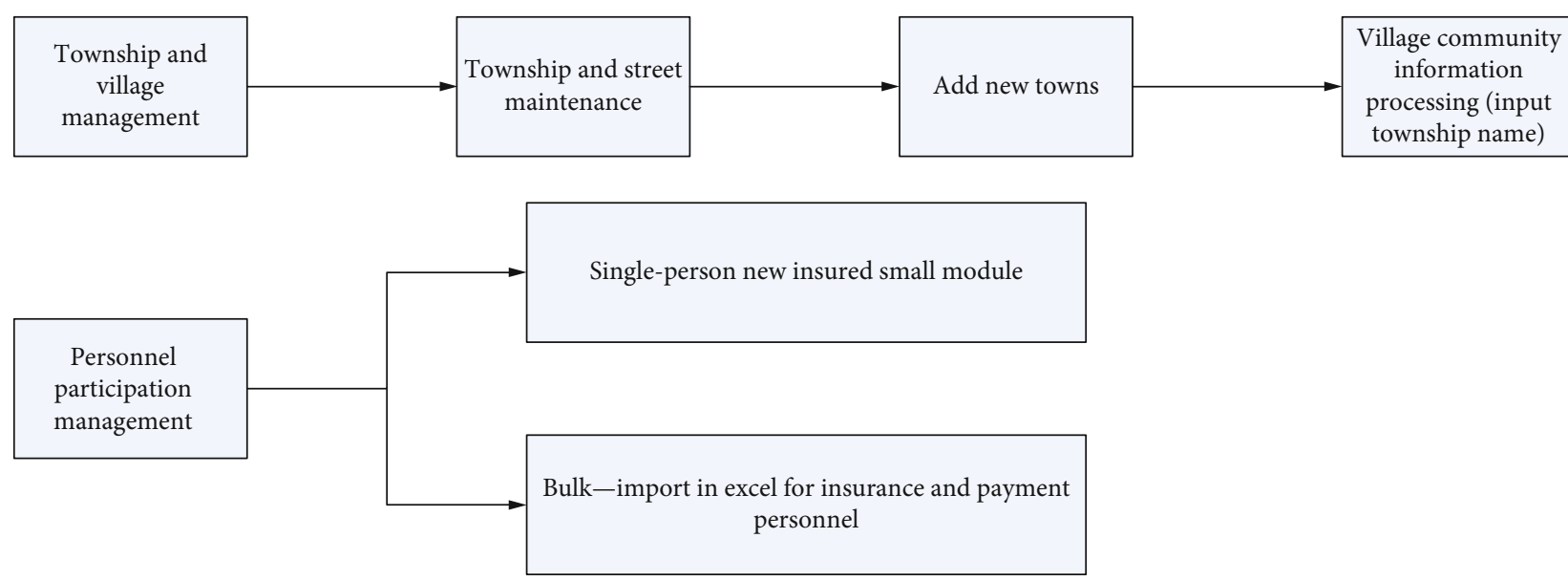

FIgURE 2: Business flow processes of insured registration.
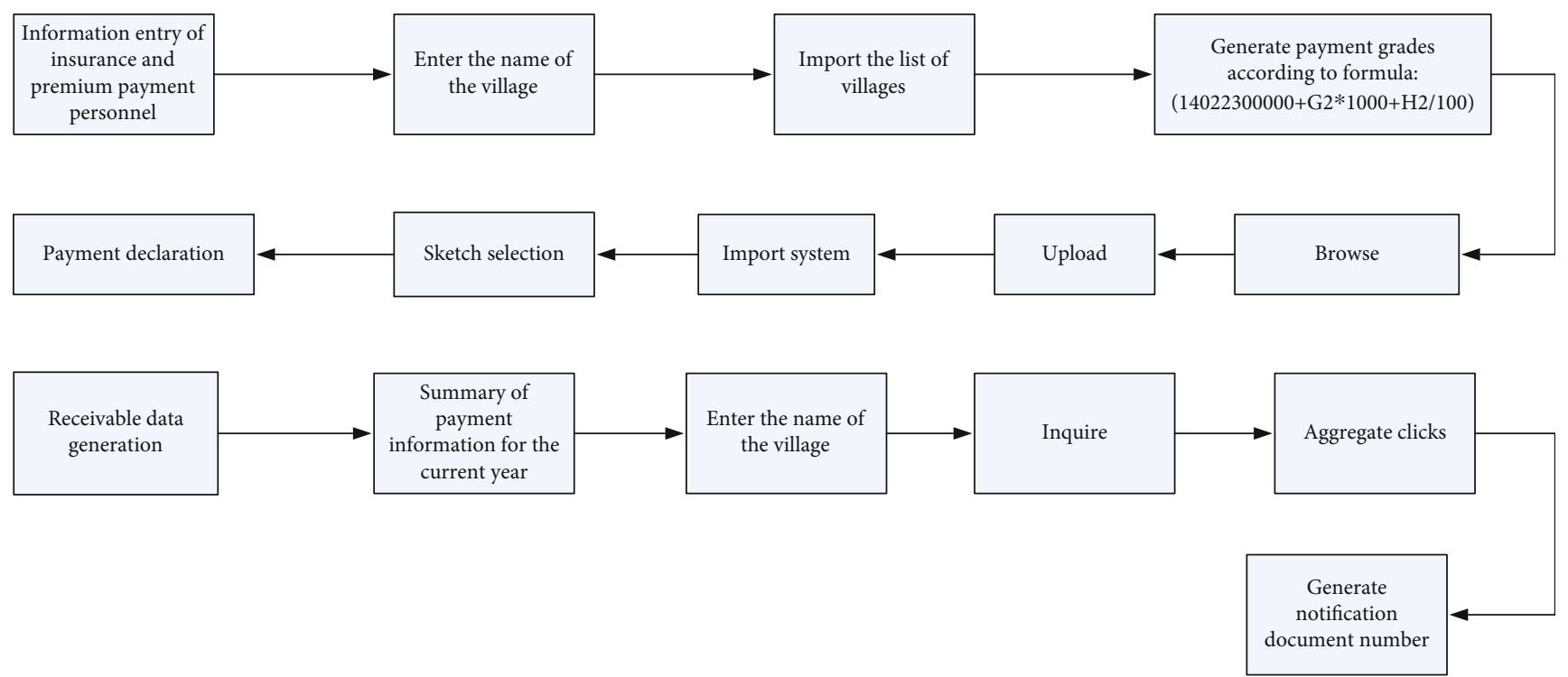

Figure 3: Business flow processes of pay insured fees.

The business flowchart of insurance premium payment is shown in Figure 3.

The flowchart of the payment of benefits is shown in Figure 4.

The above is the basic business flowchart of the system design of this article.

\section{Experimental Results of the Design and Implementation of the Rural Social Security System Based on Deep Learning}

4.1. Survey Content and Sample Analysis. The survey adopts a random sampling method. The survey content mainly covers three aspects. First, the production and living conditions of farmers, from the perspective of farmland area, income sources, main expenditures, and difficulties faced by farmers in production and life. The second is to investigate the imple- mentation status of the rural social insurance system from the perspectives of farmers' participation in the new rural social endowment insurance and new rural cooperative medical insurance, payment levels, and policy satisfaction. Third, the evaluation of the implementation effect of the rural minimum living security system is carried out from the perspectives of farmers' satisfaction with the system, the minimum living security standard, and the effect of poverty reduction. The samples involved 2 counties and cities, 7 towns, and 17 villages, and a total of 192 valid samples were collected. The sample distribution is shown in Table 1.

In the survey sample, males accounted for $61.4 \%$, and females accounted for $38.6 \%$; illiterate accounted for $16.6 \%$ (more left-behind elderly in the survey area), $41.5 \%$ of the education level is only elementary school, $31.1 \%$ of the education level is junior high school, and the education level is high school (middle school). Those with a college degree or above accounted for $6.2 \%$; those with a college degree or 

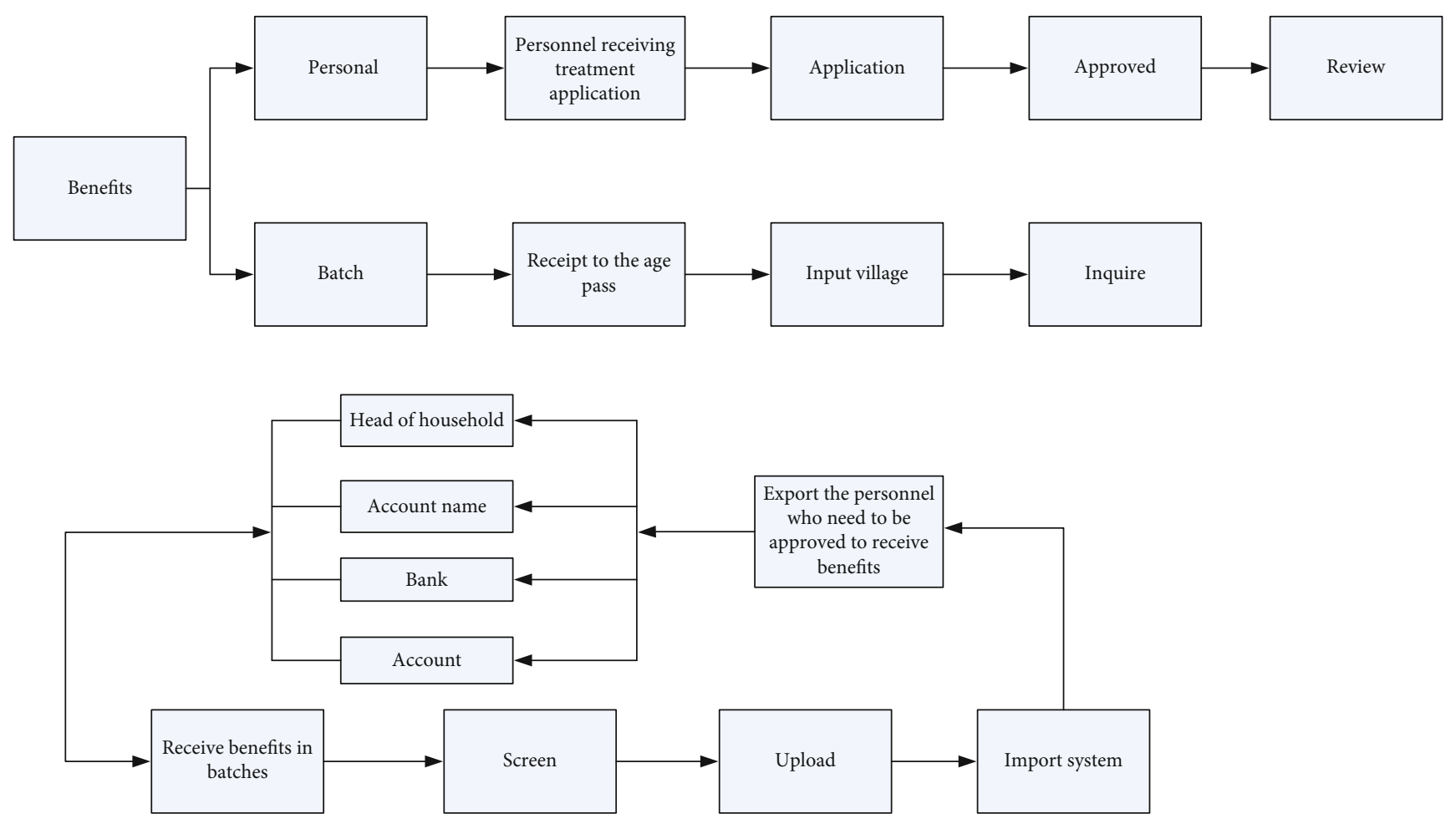

FIgURE 4: Business flow processes of benefit distribution.

above accounted for $4.1 \%$; those aged 18-29 accounted for $5.2 \%$, those aged $30-44$ accounted for $24.0 \%$, those aged $45-$ 59 accounted for $38.0 \%$, those aged $60-74$ accounted for $28.1 \%$, and those aged 75 or above accounted for $4.7 \%$; $18.8 \%$ of households had 3 persons or less, $23.4 \%$ had 4 persons, $25.0 \%$ had 5 persons, $22.4 \%$ had 6 people, and $10.4 \%$ had 7 or more people; people with financial resources of 0 accounted for $2.6 \%, 1$ person accounted for $16.1 \%, 2$ people accounted for $36.5 \%, 3$ people accounted for $30.2 \%$, 4 people accounted for $9.9 \%$, and those with 5 or more people accounted for $4.7 \%$; $28.1 \%$ indicated those with capable relatives at home, $71.9 \%$ indicated those with no capable relatives, $17.7 \%$ indicated those with college students at home, and $82.3 \%$ indicated those without college students. Accounting for $49.0 \%, 51.0 \%$ indicated those who did not go out to work; $3.3 \%$ of the cultivated land area was less than $1 \mathrm{mu}$, and $11.5 \%$ accounted for 1-2 acres (including 1, less than 2) and 2-3 acres (including 2, less than 3). Accounting for $46.5 \%, 3-4$ acres (including 3, less than 4) accounted for $23.0 \%, 4-5$ acres (including 4 , less than 5) accounted for $14.8 \%$, and 5 acres and above accounted for $24.6 \%$.

Among them, the composition of the income sources of statistical farmers is shown in Table 2.

Due to the fragility characteristics of farmers, there are many life pressures, including irresistible natural risks, lack of production materials and technological limitations, people's livelihood issues closely related to life, and systemic risks from changes in national policies. Figure 5 shows the life and production pressure of the survey sample farmers.

From the specific survey data, in the survey sample population, $12.26 \%$ of the farmers feel that the pressure on production and life is very high, $40.47 \%$ of the farmers feel that
TABLE 1: Survey sample distribution table.

\begin{tabular}{cccc}
\hline & Town & Village & Residents \\
\hline \multirow{4}{*}{ County seat A } & a & 1 & 11 \\
& b & 2 & 28 \\
& c & 2 & 25 \\
County seat B & d & 2 & 23 \\
& e & 2 & 24 \\
& a & 4 & 38 \\
\hline
\end{tabular}

TABLE 2: The composition of income sources of survey sample farmers.

\begin{tabular}{lc}
\hline Income type & The proportion (\%) \\
\hline Agricultural income & 23.23 \\
Self-employed income & 2.94 \\
Income from migrant workers & 58.07 \\
Transfer income & 4.76 \\
Other income & 13.00 \\
\hline
\end{tabular}

the pressure on production and life is relatively high, $37.48 \%$ of the farmers feel that the pressure on production and life is average, and $8.97 \%$ of the farmers feel that the pressure on production and life is very small, and the number of farmers who choose not to be pressured is 0 . It can be seen that the farmers in the survey area feel that the pressure on production and life is very high, and the relatively large samples account for $51.76 \%$ of the total sample. 


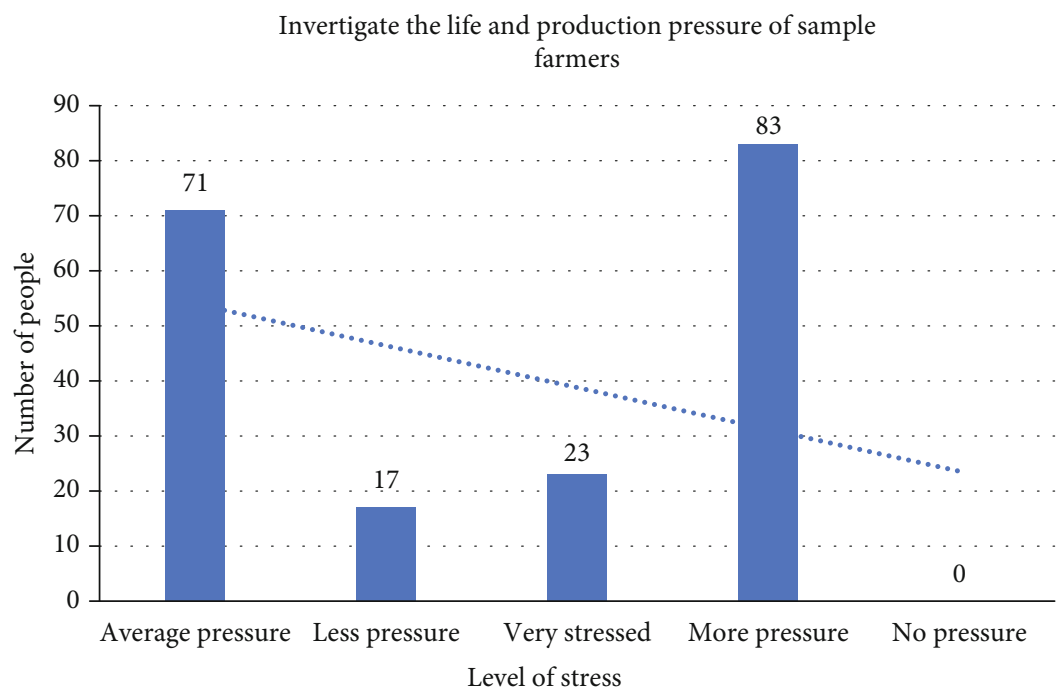

Number of people

Figure 5: Investigate the life and production pressure of sample farmers.

4.2. Farmers' Risk Aversion Methods. In the survey data, a large number of the rural surplus labor force in the survey sample source areas a and b transferred to the cities. For the sample farmers surveyed, $51 \%$ of the farmers did not receive a more stable, flexible, and higher income than those engaged in agricultural production. There are migrant workers in the family. This kind of higher income through the process of migrant workers is one of the main ways for farmers to prevent risks; the rural social security system is gradually improved in the survey area, and farmers pass on part of the risk to the society by participating in social insurance. At present, the participation rate of rural households is relatively high. The participation rate of the new rural cooperative medical system in the survey sample is as high as $98.57 \%$, and the participation rate of the new rural social endowment insurance reaches $85.66 \%$. At the same time, a small number of rural households whose income is lower than the local minimum living standard also receive subsistence allowances and will avoid falling into extreme poverty; the surveyed farmers are generally not well-educated. Even if the cost of education continues to rise, farmers are paying more and more attention to their children's education, especially their children's higher education. $23.8 \%$ of the survey sample farmer households have college students; with the in-depth development of the rural market economy, the number of farmers engaged in business activities such as self-employed businesses has gradually increased. The survey sample has 20 farmers' families engaged in large-scale business activities such as vegetables and fruits through contracted land, and 18 of the farmer households are engaged in self-employed business activities, accounting for $19.79 \%$ of the sample population; farmers participating in commercial insurance are also gradually increasing, accounting for $35.21 \%$ of the sample population. Farmers have participated in agricultural insurance and other commercial insurance, as shown in Table 3.
TABLE 3: Investigate the risk aversion portfolio of sample farmers.

\begin{tabular}{lcc}
\hline & Rate (\%) & Motivation \\
\hline Shift labor & 51 & Get higher income \\
Self-employed & 35.21 & \\
Investment education & 23.9 & Reserve human capital \\
Bank savings & 39.1 & Prevent risks \\
Pension & 85.66 & Pass on risk \\
Medical insurance & 98.57 & \\
Business insurance & 27.97 & \\
\hline
\end{tabular}

Join the satisfaction evaluation system (satisfaction evaluation is based on whether the service recipient's needs are met as an objective evaluation scale, and it is a method of final quality evaluation), as shown in Table 4.

The same as farmers' satisfaction with the new rural cooperative medical system, the survey of farmers' satisfaction with the new rural insurance (pension insurance is an important part of the social security system, and it is one of the most important types of insurance in the five major types of social insurance; the purpose of endowment insurance is to protect the basic living needs of the elderly and provide them with a stable and reliable source of life) shows that the percentages of farmers choosing very satisfied, satisfied, average, dissatisfied, and very dissatisfied are 8.94\%, 45.53\%, 34.96\%, 8.13\%, and $2.44 \%$, respectively; it can be seen that the proportion of farmers who choose to be satisfied is the largest, and more than $10.0 \%$ of farmers choose to be dissatisfied or very dissatisfied. Investigate the factors that farmers worry about participating in the new rural insurance, and the questionnaire options can also be set to multiple choices. The survey results show that $29.27 \%$ of the farmers think that the individual payment for participating in the new rural insurance is higher; $26.02 \%$ of the farmers believe that they do not understand 
TABLE 4: Satisfaction of rural households' social insurance system.

\begin{tabular}{lccccc}
\hline & Very satisfied & Satisfied & Generally satisfied & Dissatisfied & Very dissatisfied \\
\hline NCMS & 16.26 & 35.77 & 40.65 & 6.50 & 0.81 \\
New rural insurance & 8.94 & 45.53 & 34.96 & 8.13 & 2.44 \\
\hline
\end{tabular}

NCMS: new cooperative medical system.

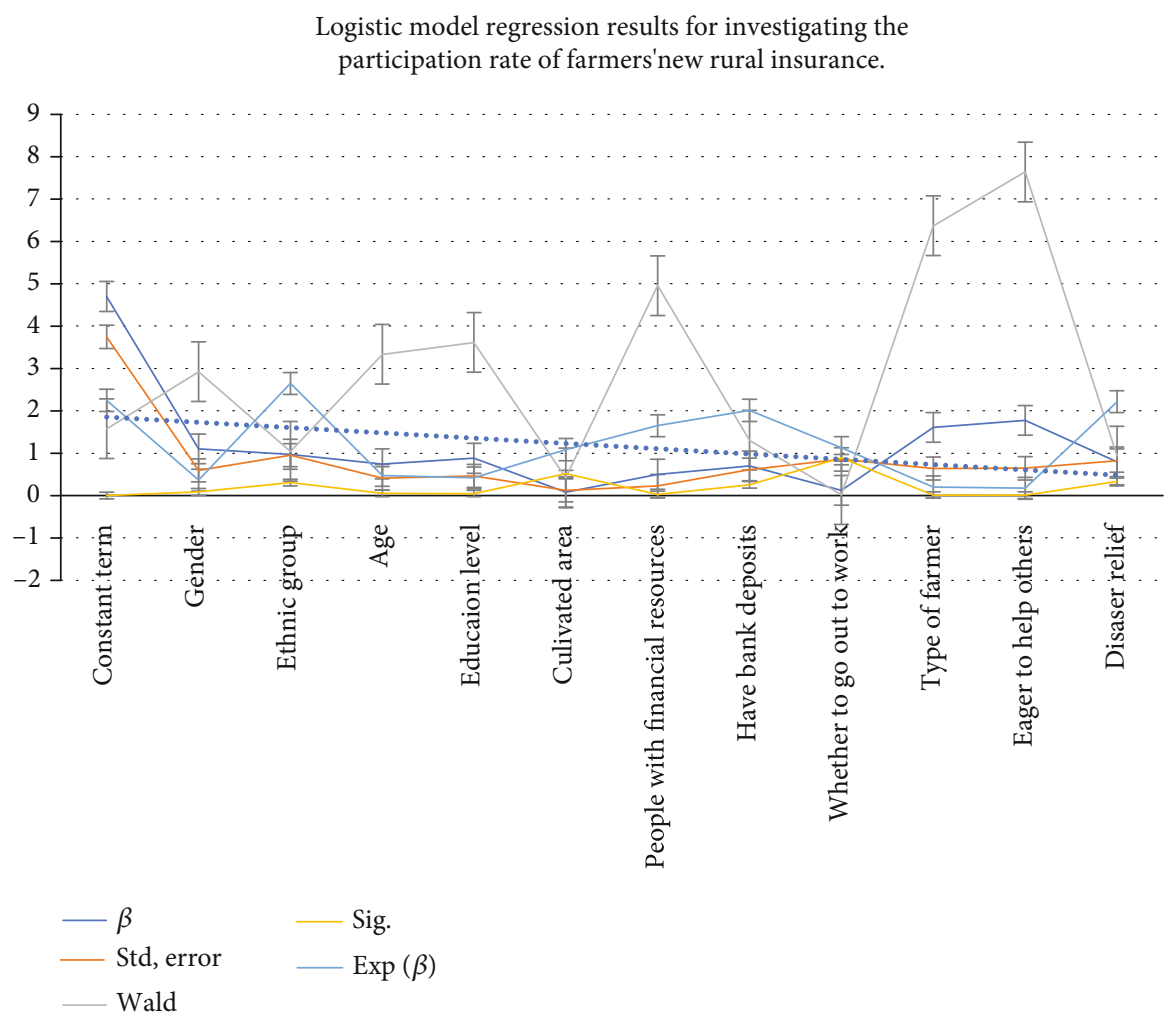

FIGURE 6: Logistic model regression results for investigating the participation rate of farmers' new rural insurance.

the new rural insurance system; $9.76 \%$ of the farmers believe that it is unnecessary to pay for the new rural insurance; $22.76 \%$ of farmers choose to rely on themselves or their children in the future; $27.64 \%$ of farmers think that the system is unstable.

4.3. Use the Logistic Model Established by the Gradient Descent Method Based on Deep Learning to Analyze the Regression Results. According to the content of the questionnaire survey, the factors that affect farmers' participation in the new rural insurance are summarized into individual characteristics of farmers, family resource endowments, and social environmental factors. Among them, the individual characteristics of farmer households include four variables: gender, ethnicity, age, and educational level; family resource endowment factors include the following: arable land area, number of households with financial resources, whether the family has bank deposits, whether the family has migrant workers, and the main income of the household (source type: agriculture, work, and business); social environmental factors include the following: whether relatives and friends give help when encountering difficulties and encountering natural disasters and whether they receive government assistance in times of difficulty. Using SPSS 19.0 statistical software, the logistic regression model established above was used to estimate the factors affecting the farmers participating in the new rural insurance in the dependent variable survey area, as shown in Figure 6.

From the overall model estimation results, the chisquared test value of the model is 29.633 (the chi-squared test is a very versatile method of hypothesis testing; its application in the statistical inference of classified data includes the following: the chi-squared test comparing two rates or two constituent ratios and comparing multiple rates or multiple constituent ratios and the chi-squared test and correlation analysis of classification data), and its corresponding Sig value is 0.002 ( $\mathrm{Sig}$ is a significant index, where generally greater than 0.05 indicates that the difference is not significant, less than 0.05 indicates that the difference is significant, and less than 0.01 indicates that the difference is extremely significant), which has reached significance, indicating that the overall estimation effect of the regression model is better. However, in terms of the effect of explaining the dependent variable for a single independent variable, the independent 


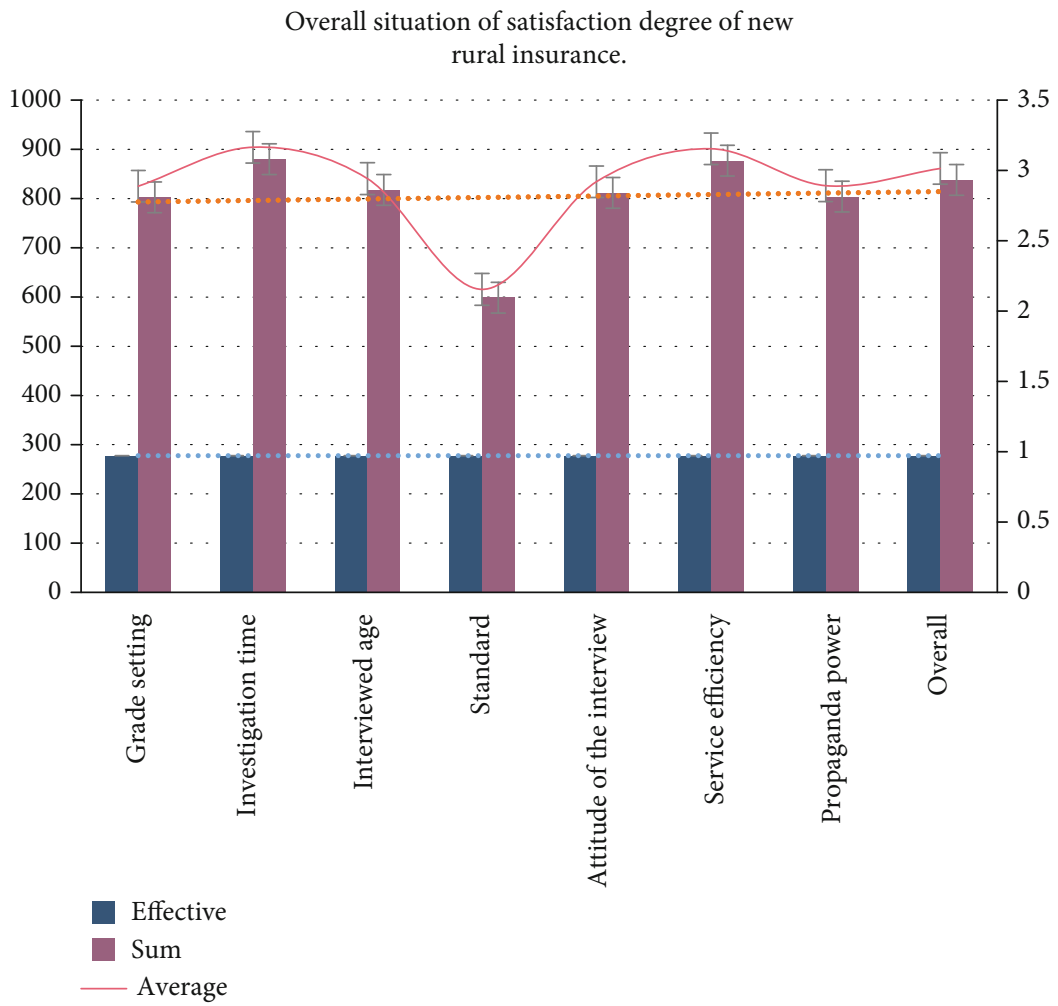

Figure 7: Overall situation of the satisfaction degree of new rural insurance.

variables in the research question are only $X_{3}, X_{4}, X_{6}, X_{9}$, and $X_{10}$. These variables are statistically significant, and the other 6 independent variables are statistically significant. The above is not significant.

From the regression model, gender and ethnicity have no significant effect on the new rural insurance participation rate of farmers in the survey area, age has a positive effect on the insurance participation rate, the education level has a negative effect on the insurance participation rate, and family resource factors have economic sources. The number of people has a positive impact on the participation rate of the new rural insurance, and whether or not they receive assistance when encountering difficulties such as natural disasters has no significant impact on the participation rate.

Finally, we have done satisfaction statistics on the rural social security system designed in this article. In the overall analysis of satisfaction with the new system, the questionnaire uses a 5-level Likert scale, with a total of 1, 2, 3, 4, and 5 ratings, which are very dissatisfied, dissatisfied, fair, satisfied, and very satisfied. The lowest score is 1 point, and the highest score is 5 points. The higher the score, the higher the satisfaction level, and vice versa. Use SPSS 24.0 software (SPSS (Statistical Product and Service Solutions) software) (initially, the full name of the software was "Statistical Software Package for Social Sciences," but with the expansion of SPSS product services and the increase in service depth, SPSS officially changed its English name to "Statistical Product and Service Solutions" in 2000, which marks that major adjustments are being made to the strategic direction of SPSS; SPSS is the general term for a series of software products and related services for statistical analysis operations, data mining, predictive analysis, and decision support tasks launched by IBM; there are Windows and Mac OS X versions) to analyze the average satisfaction data of the recovered new system. The specific distribution is shown in Figure 7.

It can be seen from the figure that the overall satisfaction rate of this questionnaire is relatively qualified, reaching an average of 2.5 for general satisfaction. Among them, the average satisfaction of the grade setting, receiving age, payment method, and service efficiency is slightly less. Higher than average, it shows that some rural residents are quite satisfied with these tasks, but the proportion is not too large. It shows that the design of the rural social security system based on deep learning designed in this paper is relatively successful.

\section{Conclusions}

The research design shows that the design of the rural social security system based on deep learning proposed in this article is more powerful in statistical data, better in data retention, and more timely in response to the system than other current rural social security systems. The statistical capacity is also more powerful. This article uses the sample collection method and statistical analysis method, collects samples, simplifies the algorithm, and establishes a new rural social security system. The data collected by the system shows that the proportion of farmers who choose very satisfied, satisfied, average, dissatisfied, and very dissatisfied with the satisfaction of the new rural insurance is $8.94 \%, 45.53 \%, 34.96 \%$, $8.13 \%$, and $2.44 \%$. It can be seen that the proportion of 
farmers who choose to be satisfied is the largest, and more than $10.0 \%$ of farmers choose to be dissatisfied or very dissatisfied. Investigate the factors that farmers worry about participating in the new rural insurance, and the questionnaire options can also be set to multiple choices. The survey results show that $29.27 \%$ of the farmers think that the individual payment for participating in the new rural insurance is higher; $26.02 \%$ of the farmers believe that they do not understand the new rural insurance system; $9.76 \%$ of the farmers believe that it is unnecessary to pay for the new rural insurance; $22.76 \%$ of farmers choose to rely on themselves or their children in the future; $27.64 \%$ of farmers think that the system is unstable. The data collected by the system designed in this paper is very accurate, the sample size is large enough, and the automatic analysis of the system is also very fast. The shortcomings of this article are as follows: the preliminary work is very complicated and cumbersome, and the part related to semantic calculation is relatively obscure and cannot be widely applied to other system designs. Due to the limitation of conditions, this paper only selected two counties and cities in the north for data collection and did not comprehensively consider the development trend of rural areas in the north and south of my country. This system cannot be used in rural social security throughout the country. In the simplification and refinement of the future system, we can comprehensively select rural areas in different situations in my country's southern, northern, and even western regions for larger data analysis so that the rural social security system based on deep learning can be representative across the country.

\section{Data Availability}

No data were used to support this study.

\section{Conflicts of Interest}

The author declares no conflict of interest.

\section{Acknowledgments}

This work was supported by Scientific research projects in higher education institutions of Inner Mongolia (NJSY18029).

\section{References}

[1] T. Wu, S. Wen, S. Liu et al., "Detecting spamming activities in Twitter based on deep-learning technique," Concurrency and Computation: Practice and Experience, vol. 29, no. 19, pp. 14-17, 2017.

[2] J. Chen and S. Smys, "Social multimedia security and suspicious activity detection in SDN using hybrid deep learning technique," Journal of Information Technology and Digital World, vol. 2, no. 2, pp. 108-115, 2020.

[3] M. Zhong, Y. Zhou, and G. Chen, "A security log analysis scheme using deep learning algorithm for IDSs in social network," Security and Communication Networks, vol. 2021, no. 4, 2021.

[4] K. Huang, Q. Li, and G. Zhu, "Electric tower fault detection method based on deep learning," IPPTA: Quarterly Journal of Indian Pulp and Paper Technical Association, vol. 30, no. 8, pp. 733-737, 2018.

[5] R. Greiner, L. Fernandes, F. McCartney, and J. Durante, "Reasons why some irrigation water users fail to comply with water use regulations: a case study from Queensland, Australia," Land Use Policy, vol. 51, no. 9, pp. 26-40, 2016.

[6] C. Kumar, T. S. Bharati, and S. Prakash, "Online social network security: a comparative review using machine learning and deep learning," Neural Processing Letters, vol. 53, no. 1, pp. 843-861, 2021.

[7] D. Osabe, Y. Litsuka, and A. Higashi, "Solutions to social problems leveraging image analysis technology based on machine learning," Fujitsu Scientific \& Technical Journal, vol. 53, no. 3, pp. 32-38, 2017.

[8] C. Zhou, F. Li, W. Cao, C. Wang, and Y. Wu, "Design and implementation of a novel obstacle avoidance scheme based on combination of CNN-based deep learning method and liDAR-based image processing approach," Journal of Intelligent and Fuzzy Systems, vol. 35, no. 2, 2018.

[9] A. Dayal, N. Paluru, L. R. Cenkeramaddi, J. Soumya, and P. K. Yalavarthy, "Design and implementation of deep learning based contactless authentication system using hand gestures," Electronics, vol. 10, no. 2, pp. 182-184, 2021.

[10] M. Saber and E. M. Elkenawy, "Design and implementation of accurate frequency estimator depend on deep learning," International Journal of Engineering \& Technology, vol. 9, no. 2, 2020.

[11] W. Qian and Y. Ge, "The implementation of leisure tourism enterprise management system based on deep learning," International Journal of Systems Assurance Engineering and Management, vol. 2, no. 9, pp. 45-51, 2021.

[12] A. T. Q. Al-Aqbi, R. R. K. Al-Taie, and S. K. Ibrahim, "Design and implementation of online examination system based on MSVS and SQL for university students in Iraq," Webology, vol. 18, no. 1, pp. 416-430, 2021.

[13] C. Wang, L. Zheng, B. Li, and Z. Li, "Design and implementation of EtherCAT Master based on Loongson," Procedia Computer Science, vol. 183, no. 2, pp. 462-470, 2021.

[14] O. Akanbi and O. Abegunde, "Design and implementation of mobile information system for Federal Road Safety Corps (FRSC) of Nigeria," International Journal of Sensor Networks and Data Communications, vol. 9, no. 1, 2021.

[15] S. Dario Alessandro et al., "Mobile-edge computing architecture: the role of MEC in the Internet of Things," IEEE Consumer Electronics Magazine, vol. 5, no. 4, pp. 84-91, 2016.

[16] P. Corcoran and S. K. Datta, "Mobile-edge computing and the Internet of Things for consumers: extending cloud computing and services to the edge of the network," IEEE Consumer Electronics Magazine, vol. 5, no. 4, pp. 73-74, 2016.

[17] Z. Tian, D. Liu, W. Chen, W. Wang, S. Pan, and N. Guo, "Analysis and optimization of asymmetric wireless power transfer in concrete," Wireless Communications and Mobile Computing, vol. 2020, no. 3, 2020.

[18] V. Pande, C. Marlecha, and S. Kayte, "A review-fog computing and its role in the Internet of Things," International Journal of Engineering Research and Applications, vol. 6, no. 10, pp. 2248-96227, 2016.

[19] X. Sun and N. Ansari, "EdgeIoT: mobile edge computing for the Internet of Things," IEEE Communications Magazine, vol. 54, no. 12, pp. 22-29, 2016. 
[20] H. Ning, Y. Li, F. Shi, and L. T. Yang, "Heterogeneous edge computing open platforms and tools for Internet of Things," Future Generation Computer Systems, vol. 106, no. 11, pp. 67-76, 2020.

[21] D. Thanh, K. Younghan, and L. Hyukjoon, "A location-based interactive model of Internet of Things and cloud (IoT-cloud) for mobile cloud computing applications," Sensors, vol. 17, no. 3, pp. 489-491, 2017.

[22] N. Ansari and X. Sun, "Mobile edge computing empowers Internet of Things," IEICE Transactions on Communications, vol. 101, no. 3, pp. 604-619, 2018.

[23] A. Kamilaris and A. Pitsillides, "Mobile phone computing and the Internet of Things: a survey," IEEE Internet of Things Journal, vol. 3, no. 6, pp. 885-898, 2017.

[24] O. Fragou and A. Mavroudi, "Exploring Internet of Things, mobile computing and ubiquitous computing in computer science education: a systematic mapping study," International Journal of Technology in Education and Science, vol. 4, no. 1, pp. $72-85,2020$.

[25] X. Lyu, H. Tian, L. Jiang et al., "Selective offloading in mobile edge computing for the green Internet of Things," IEEE Network, vol. 32, no. 1, pp. 54-60, 2018.

[26] P. Botu, "Strong semantic computing," Procedia Computer Science, vol. 123, no. 7, pp. 98-103, 2018, Conversations with the following people helped me develop some aspects of this paper: Riccardo Sanz, Jack Copeland, John Barker, Kevin $\mathrm{O} \backslash$ Regan and Gadi Pinkus.

[27] L. Fabisiak, "Web service usability analysis based on user preferences," Journal of Organizational and End User Computing, vol. 30, no. 4, pp. 1-13, 2018.

[28] T. Grubljesic, P. S. Coelho, and J. Jaklic, "The shift to socioorganizational drivers of business intelligence and analytics acceptance," Journal of Organizational and End User Computing, vol. 31, no. 2, pp. 37-64, 2019. 